\title{
3D Bioprintability of Konjac Glucomannan Hydrogel
}

\author{
Yulong WANG, Lu HAN*, Jis YAN, Kun HU, Luhai LI, Huai ZHANG, Hao AI \\ Beijing Engineering Research Center of Printed Electronics, Beijing Institute of Graphic Communication, Beijing 102600, \\ China \\ crossref http://dx.doi.org/10.5755/j01.ms.26.1.20336
}

Received 12 March 2018; accepted 24 July 2018

\begin{abstract}
Konjac glucomannan has potential applications in bio-printing, due to its unique properties, such as high viscosity, waterholding capacity and easy gelatinization. In this study, the rheological properties, i.e. the viscosity with changing the shear rate and the storage modulus $G$ ' and loss modulus $G$ ' curve, of different concentrations of konjac gum hydrogel, were thoroughly measured. Furthermore, the pore sizes of various concentrated konjac gum hydrogel were observed under scanning electron microscopy (SEM). The bio-printability of konjac gum hydrogel was thoroughly evaluated using pistontype 3D bio-printer. It was showed that, $7 \%$ konjac gum hydrogel demonstrated the best bio-printability, which has potentially applied as scaffold materials in bio-printing field.

Keywords: konjac glucomannan, hydrogel, 3D bio-printability.
\end{abstract}

\section{INTRODUCTION}

$3 \mathrm{D}$ bio-printing is a technique that uses $3 \mathrm{D}$ printer to distributing bio-active ingredients, including protein, cells and some nutrients on the support materials to create 3D tissue constructs $[1,2]$. Bio-printing research has rapidly developed over the past decade and is widely used in tissue engineering [3]. The scaffold materials for bio-printing should meet these characteristics including biocompatibility [4,5], biodegradability [6], high structural fidelity and excellent printability $[2,7]$. Hydrogels as predetermined candidates are well positioned to solve these problems.

Hydrogels are a class of hydrophilic or amphiphilic three-dimensional network polymers with chemically or physical cross-linked structure [8,9]. They are structurally similar to native extracellular matrices (ECM) [10]. They can also provide three dimensional structures for cell adhesion, proliferation [11], and transportation of cytokines, nutrients and metabolic waste [12]. Therefore, it is widely used as scaffold materials for bio-printing application. The hydrogels currently used for bio-printing can be divided into synthetic hydrogels and natural hydrogels [13]. Compared to the former, natural hydrogels, e.g. gelatin [14], sodium alginate [15, 16], chitosan [17] and konjac glucomannan [18], are not only rich in natural world, but also have lower toxicity, good biodegradability [19] and suitable viscosity for bio-printing [20]. Konjac glucomannan (KGM) is a natural polysaccharide consisting of $\beta$-D-glucose and $\beta$-Dmannose bound by $\beta-1,4$ glycosidic bonds at a molar ratio of 1:1.6 [21, 22]. KGM has unique properties, such as high viscosity, water-holding capacity and easy gelatinization [23], which make it widely applied in drug delivery [24, 25], food [26] and also chemical engineering [27]. However, there are few studies on its 3D bio-printability. Therefore, it is necessary to study its bio-printability of KGM with varing concentration.
In this study, the viscosity and their shear thinning behavior of $4 \%, 5 \%, 6 \%, 7 \%$ and $8 \%$ of KGM hydrogel were detected. The microstructure of freeze-dried KGM hydrogel was observed using scanning electron microscope (SEM). The bioprintability of KGM with different concentration was then thoroughly evaluated.

\section{MATERIALS AND METHODS}

\subsection{Materials}

Konjac Glucomannan (CKAA2030, $95 \%$ ) was purchased from Chengdu Sheli Konjac Science Plantation Processing Park Co., Ltd. Fab@ home 3D printer. (Model 3, Seraph Robotics Inc., NY, USA. The Fab@Home is a syringe-based deposition system. An X-Y-Z gantry system moves a syringe pump across a $20 \times 20 \times 20 \mathrm{~cm}$ $(7.87 \times 7.87 \times 7.87 \mathrm{inch})$ build volume at a maximum speed of $10 \mathrm{~mm} / \mathrm{s}$ and resolution of $25 \mu \mathrm{m}$. Multiple syringes can be controlled independently to deposit material through syringe tips. The syringe displacement could be controlled with a microliter precision.

\subsection{Hydrogel preparation}

$4 \%, 5 \%, 6 \%, 7 \%$ and $8 \%(w / v)$ of KGM hydrogels were obtained by dissolving $4 \mathrm{~g}, 5 \mathrm{~g}, 6 \mathrm{~g}, 7 \mathrm{~g}$ and $8 \mathrm{~g}$ of konjac flour in $100 \mathrm{ml}$ deionized water at room temperature, respectively. The mixtures were stirred for 30 minutes and then placed in refrigerator overnight at $4{ }^{\circ} \mathrm{C}$ for subsequent experiment.

\subsection{Rheological properties of KGM hydrogel}

The as-prepared KGM hydrogels were centrifuged to eliminate air. The viscosities with changing the shear rates, as well as the changes of the storage modulus $G^{\prime}$ and the loss modulus $G$ ', with varying the frequency were measured using the TA2000ex Rheometer. An aluminum plate with

\footnotetext{
* Corresponding author. Tel.: 13120410616.

E-mail address: hanlu@iccas.ac.cn (L. Han)
} 
diameter of $40 \mathrm{~mm}$ was used at a distance of $1 \mathrm{~mm}$ between the plate and the sample stage. In the test, the shear rate and the angular frequency were set from 0.01 to $100 \mathrm{~s}^{-1}$ and 0.1 to $100 \mathrm{rad} / \mathrm{s}$, respectively.

\subsection{Morphological characterization of KGM hydrogel}

The as-prepared konjac hydrogels were pre-frozen in a $-20{ }^{\circ} \mathrm{C}$ refrigerator for 1 hour, and then placed in a LGJ$10 \mathrm{C}$ freeze dryer. After the temperature of the materials were reduced to $-60{ }^{\circ} \mathrm{C}$, vacuum was applied and freezedried to room temperature. And then the dried hydrogels were adhered to a conductive tape and gold was sprayed. The microscopic internal morphology was observed using the SU8020 scanning electron microscope (SEM, SU8020, Hitachi, Japan) at voltage $10 \mathrm{kV}$, working distance $8 \mathrm{~mm}$, magnification 60 times.

\subsection{D bio-printing scaffolds using KGM hydrogel}

The 3D scaffolds were fabricated using the Fab@home model 3D printer. At first, a 3D porous mesh model was created using Solidworks CAD, after convert the model into an STL format, the Serapstudio software was usded to layer and generate XDFL files. The KGM hydrogels were transferred to the syringes of printer, which then centrifuged at $8000 \mathrm{r} / \mathrm{min}$ to remove bubbles before the syringes were installed on the printer. Using Seraprint software to determine the initial position of the print by adjusting the print platform $\mathrm{x}$-axis, $\mathrm{y}$-axis and $\mathrm{z}$-axis, a square model $(2 \times 2 \mathrm{~cm})$ with four layers was designed as a XDFL file, which was input into $3 \mathrm{D}$ printer to fabricate the latticed square.

\subsection{Identifying 3D-printing parameters}

To optimize the printing parameters, a monolayer model containing eight line paths with $20 \mathrm{~mm}$ long was designed and constructed. The optimized parameters [28, 29] were set at nozzle diameter $0.41 \mathrm{~mm}$, path height $0.41 \mathrm{~mm}$, path width $0.41 \mathrm{~mm}$, print speed $5 \mathrm{~mm} \mathrm{~s}^{-1}$, deposition rate $0.009 \mathrm{~mm} \mathrm{~mm}^{-1}$, and pushout/suckback $0.02 \mathrm{~s}$.

\section{RESULTS AND DISCUSSION}

\subsection{Rheological analyses}

Shape fidelity of printed 3D constructs depends not only on printing parameters, but on the rheological properties of as-prepared materials as well. The prepared scaffold materials should have shear thinning characteristics, so that they have lower viscosities at the high shear rates generated during extrusion. After extrusion, viscosity increases result in high print fidelity and cell viability.

We then tested the viscosity of different concentrated konjac gums with changing the shear rate. As shown in Fig. 1, the viscosity of konjac gum hydrogel decreases with the increase of shear rate, which showed false plasticity and the characteristics of shear thinning. Below the shear rate of $0.1 \mathrm{~s}^{-1}$, the konjac gum appear on the curve platform, which is attributed to that konjac gum hydrogel may produce enough new intermolecular entanglement at a lower shear rate. Overall, the viscosity of konjac gums hydrogel increases with increasing their concentration. Moreover, at a relatively high level of shear rate, their viscosities tend to be decreased. However, if the viscosity of hydrogel is too high, it is difficult for the extrusion. Thus the increasing shear stress is a damage to cell viability. These results give us a hint; it is very important to choose a suitable concentration of konjac gum hydrogel for printing jobs.

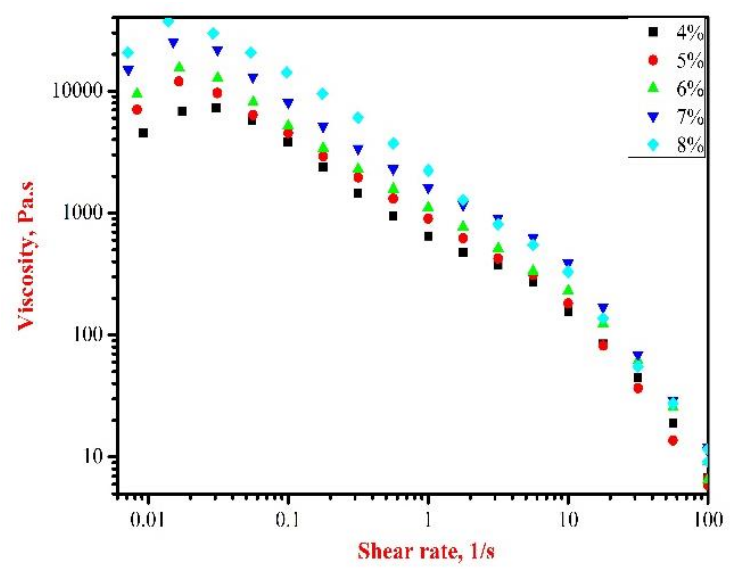

Fig. 1. The shear thinning properties of different concentrated konjac gums with changing the shear rates

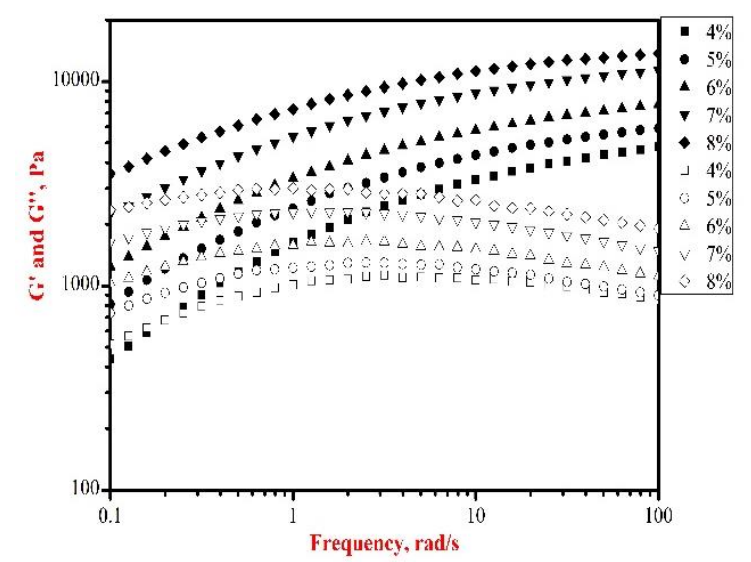

Fig. 2. Storage modulus $G$ '(solid) and loss modulus $G$ ''(hollow) of konjac hydrogels with varying the angular frequency from 0.1 to $100 \mathrm{rad} / \mathrm{s}$

The storage modulus $G^{\prime}$ and loss modulus $G^{\prime \prime}$ curve of different concentrated konjac gum hydrogel were further determined, as displayed in Fig. 2. $4 \%$ konjac gum hydrogel showed that the loss modulus $G$ ', is higher than the storage modulus $G^{\prime}$ at the lower vibration frequency, which may be due to that the hydrogel is viscous, similar to the liquid rheological behavior. Therefore, it is very hard to maintain the shape of printed structure when using $4 \%$ konjac gum hydrogel because of its strong liquidity. When the concentration of konjac gum hydrogel is higher than $5 \%$, the $G^{\prime}$ value is higher than the $G$ ' value. And also, the $G$ ' and $G$ ', values are all increasing with enhancing the concentration of konjac gum hydrogels. These results demonstrated that elastic response of these hydrogels is stronger than the viscous response. The above $5 \%$ of konjac gum hydrogel systems showed a solid-like behavior [30]. Moreover, the storage modulus $\mathrm{G}^{\prime}$ is usually considered as a measure of the extent of gel network formation [31]. The higher G' value of the gel the stronger is gel intensity [32]. In Fig. 2, we can also observe that, in the whole frequency 
range of linear viscoelastic region, the elastic behavior predominates over its viscous behavior. These features are characteristic of a hydrogel, which is useful for keeping shape fidelity in the process of 3D printing.

\subsection{Morphological characterization}

The SEM images of freeze-dried konjac gum hydrogel were recorded. In Fig. 3, the konjac gum hydrogels are porous with a three-dimensional interconnected microstructure. Furthermore, with increasing the concentration of konjac gum, their internal pore size decreases and the pore density increases. The $4 \%$ of konjac gum hydrogel displayed an average pore size of $235.3 \mu \mathrm{m} \pm 73.5 \mu \mathrm{m}$. However, the average pore sizes decreased to $191.2 \mu \mathrm{m} \pm 70.8 \mu \mathrm{m}, 116.3 \mu \mathrm{m} \pm 60.2 \mu \mathrm{m}$, $70.1 \mu \mathrm{m} \pm 32.8 \mu \mathrm{m}, 59.38 \mu \mathrm{m} \pm 33.4 \mu \mathrm{m}$ in $5 \%, 6 \%, 7 \%$ and $8 \%$ of konjac gum hydrogels, respectively. This may be ascribed to the following three factors: the density of polymer konjac polysaccharide chains, the entanglements between the chains and the increasement of intermolecular hydrogen bonding. Moreover, due to the concentration of konjac gum increasing, their inner water content decrease accordingly.


e

Fig. 3. The microscopic morphology of freeze-dried konjac gum hydrogels (a, b, c, $\mathrm{d}$ and e represent $4 \%, 5 \%, 6 \%, 7 \%$ and $8 \% \mathrm{KGM})$

Therefore, after konjac gum hydrogel being freezedried, the inner pore size decreased. Moreover, the pore size of hydrogel should meet the functions of the target tissues [33]. If pore size is too small or too large, stress is induced in the cells, which will then reduce cell viability. Because the size of human cells is usually 10 to $20 \mu \mathrm{m}$, a pore size of $50-100 \mu \mathrm{m}$ may be most suitable for cell adhesion, penetration and colonization. Hence, $7 \%$ of konjac gum gel is most appropriate for subsequent bio-printing application.

\subsection{D bio-printability evaluation of konjac gum hydrogel}

The gel was bio-printed using the fab @ home model 3 printer. A $2 \mathrm{~cm} \times 2 \mathrm{~cm}$ cube model was designed. In Fig. 4, it was shown that, the printed cube distorted using $4 \%$ of konjac gum hydrogel due to its high viscousness. While the other concentrations of konjac gum hydrogel could maintain excellent cube shape. Moreover, to further evaluate the bio-printability of konjac gum hydrogels, the lines were printed using the optimized parameters, the results of which were displayed in Fig. 5. We observed that, the printing quality of $4 \%$ and $5 \%$ konjac gum hydrogels was worse than that of $6 \%, 7 \%$ and $8 \%$ konjac gum hydrogels. For $4 \%$ and $5 \%$ konjac gum hydrogels, at the corner with right angle, the extrusion of hydrogel was doubled, which would cause the non-uniform layer height in 3D bio-printing. The error of layer height will be accumulated layer by layer until the failure of bio-printing [34]. However, for $7 \%$ and $8 \%$ konjac gum hydrogels, the phenomenon of non-uniform extrusion was not obvious. Comparatively, the corner with right angle could be obtained when using $7 \%$ of konjac gum hydrogels. This result was consistent with the conclusion that $7 \%$ of konjac gum gel is most appropriate for subsequent bio-printing application (Fig. 3).

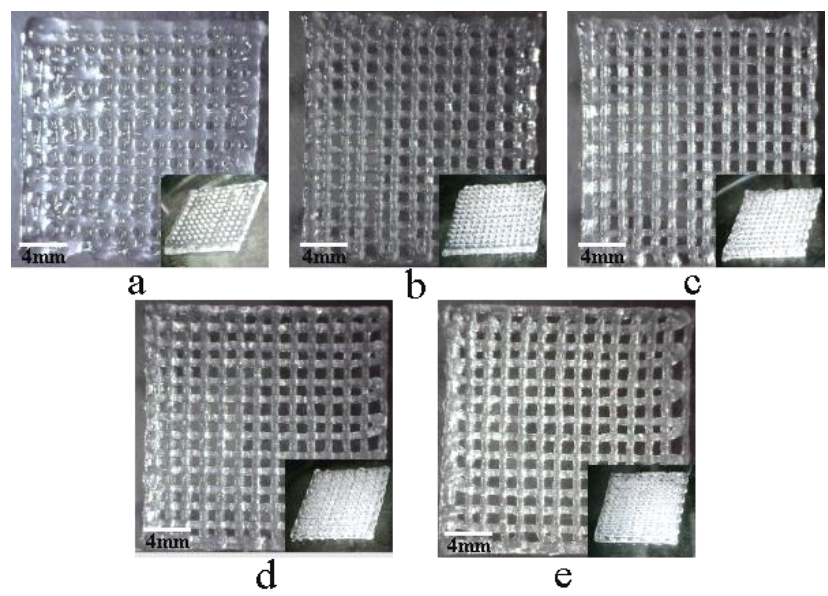

Fig. 4. Representative macroscopic images of printed KGM scaffolds (a, b, c, d and e represent $4 \%, 5 \%, 6 \%, 7 \%$ and $8 \% \mathrm{KGM})$, the scale bar is $4 \mathrm{~mm}$

Subsequently, the length and width of the printed lines were measured to further assess the bio-printability of konjac gum hydrogel, as shown in Fig. $6 a$ and b, respectively. In Fig. 6 a, the original length of printed lines was set to be $20 \mathrm{~mm}$. The average lengths of actually printed lines were all lower than the theoretical value of $20 \mathrm{~mm}$. However, for $7 \%$ konjac gum hydrogel, the length of printed lines was about $19.1 \mathrm{~mm}$, which is the nearest to the theoretical value in the five groups. In Fig. 6 b, the average widths of printed lines were all higher than the diameter of nozzle, i.e. $0.41 \mathrm{~mm}$. However, for $7 \%$ konjac gum hydrogel, the average width of printed lines was around $0.45 \mathrm{~mm}$, which is also the nearest to the nozzle diameter of $0.41 \mathrm{~mm}$ in the five groups. Therefore, we can also conclude that, $7 \%$ konjac gum hydrogel showed the best bioprintability. 


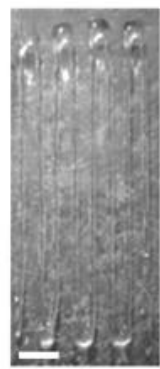

a

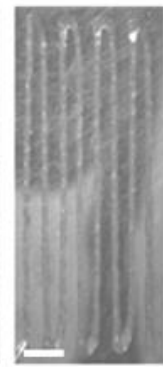

b

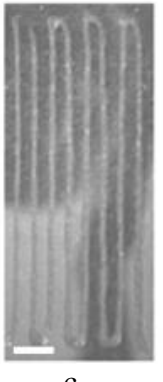

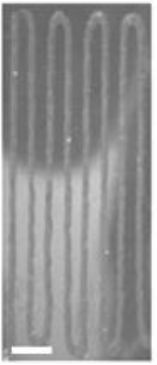

d

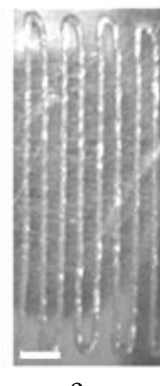

Fig. 5. Printed lines using different concentrated konjac gum with optimal printing parameters (a, b, c, d and e represent $4 \%$, $5 \%, 6 \%, 7 \%$ and $8 \% \mathrm{KGM}$ ), the scale bar is $2 \mathrm{~mm}$

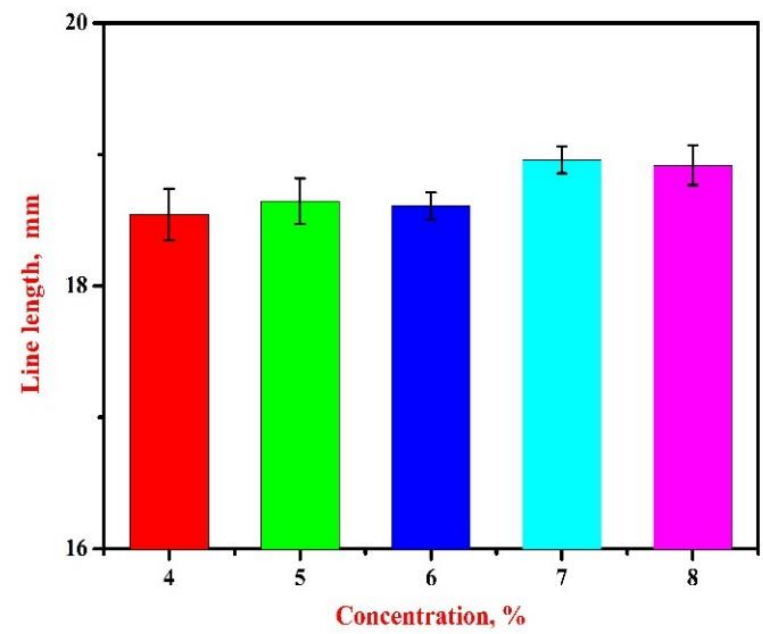

a

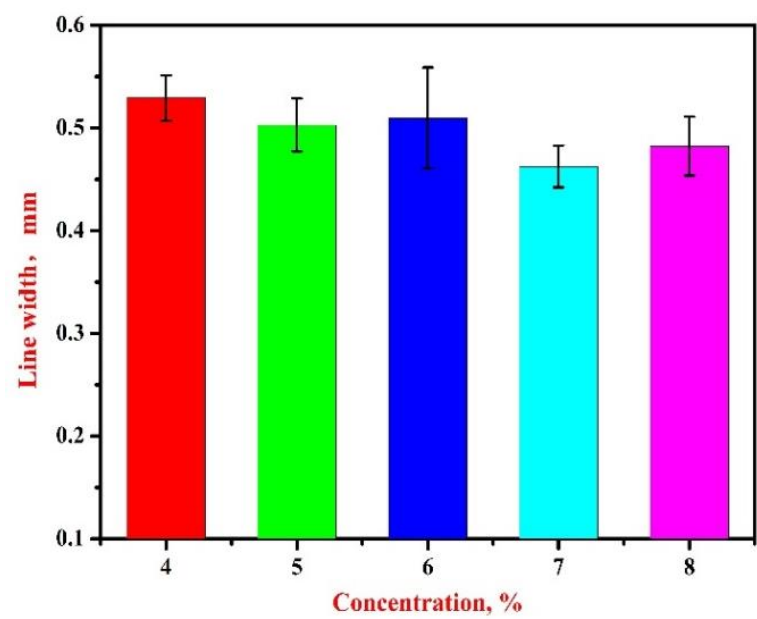

$\mathrm{b}$

Fig.6. $\mathrm{a}$ - lengths of printed lines; $\mathrm{b}$ - widthsof printed lines

\section{CONCLUSIONS}

In conclusion, it was shown that different concentration of konjac gum have shear thinning characteristics, which is advantageous for the extrusion of bioprinters, and when the concentration of konjac glucomannan is higher than $4 \%$, the elastic modulus is higher than the loss modulus over the entire test range, which is useful for keeping shape fidelity in the process of 3D printing. The pore size of $7 \%$ konjac gum hydrogel was $70.1 \mu \mathrm{m} \pm 32.8 \mu \mathrm{m}$, which is most suitable for cell adhesion, penetration and colonization. Moreover, comparatively, $7 \%$ konjac gum hydrogel could best maintain the shape of printed structure. By evaluating the length and width of the printed lines, $7 \%$ of konjac gum hydrogel has the best printability, which is most appropriate for subsequent bio-printing application.

\section{Acknowledgments}

This work was financially supported in part by Beijing Municipal Commission of Education Project (Grant No. KM201810015002), a project founded by Beijing Institute of Graphic Communication (Grant No. Ea201802), a foundation for research of great general character key technology for thin-film printed electronics (Grant No. 04190117013), and a grant from Beijing collaborative innovation for green printing and publication.

\section{REFERENCES}

1. Mironov, V., Reis, N., Derby, B. Review: Bioprinting: A Beginning Tissue Engineering 12 (4) 2006: pp. 631-634. https://doi.org/10.1089/ten.2006.12.631

2. Chimene, D., Lennox, K.K., $\quad$ Kaunas, R.R., Gaharwar, A.K. Advanced Bioinks for 3D Printing: A Materials Science Perspective Annals of Biomedical Engineering 44 (6) 2016: pp. 2090-2102. https://doi.org/10.1007/s10439-016-1638-y

3. Yu, S.Z., Kan, Y., Aleman, J., MollazadehMoghaddam, K., Bakht, S.M., Yang, J.Z., Jia, W.T., Dell'Erba, V., Assawes, P., Shin, S.R., Dokmeci, M.R., Oklu, R., Khademhosseini, A. 3D Bioprinting for Tissue and Organ Fabrication Annals of Biomedical Engineering 45 (1) 2017: pp. $148-163$. https://doi.org/10.1007/s10439-016-1612-8

4. Jose, R.R., Rodriquez, M.J., Dixon, T.A., Omenetto, F.G., Kaplan, D.L. Evolution of Bioinks and Additive Manufacturing Technologies for 3D Bioprinting Acs Biomaterials Science \& Engineering $2(10)$ 2016: pp. $1662-1678$. https://doi.org/10.1021/acsbiomaterials.6b00088

5. Malda, J., $\quad$ Visser, J., $\quad$ Melchels, F.P., $\quad$ Jungst, T., Hennink, W.E., Dhert, W.A., Groll, J., Hutmacher, D.W. 25th Anniversary Article: Engineering Hydrogels for Biofabrication Advanced Materials 25 (36) 2013: pp. $5011-5028$. https://doi.org/10.1002/adma.201302042

6. Tan, H.P., Marra, K.G. Injectable, Biodegradable Hydrogels for Tissue Engineering Applications Materials 3 (3) 2010: pp. 1746-1767. https://doi.org/10.3390/ma3031746

7. Hinton, T.J., Jallerat, Q., Palchesko, R.N., Park, J.H., Grodzicki, M.S., $\quad$ Shue, H.J., $\quad$ Ramadan, M.H., Hudson, A.R., Feinberg, A.W. Three-Dimensional Printing of Complex Biological Structures by Freeform Reversible Embedding of Suspended Hydrogels Science Advances 1 (9) 2015: pp. e1500758. https://doi.org/ 10.1126/sciadv.1500758

8. Zhang, Y.S., Khademhosseini, A. Advances in Engineering Hydrogels Science 356 (6337) 2017: pp. eaaf3627. https://doi.org/10.1126/science.aaf3627

9. Thakur, S., Govender, P.P., Mamo, M.A., Tamulevicius, S., Mishra, Y.K., Thakur, V.K. Progress in Lignin Hydrogels and Nanocomposites for Water Purification: Future Perspectives Vacuum 146 2017: pp. $342-355$. https://doi.org/10.1016/j.vacuum.2017.08.011 
10. Annabi, N., Tamayol, A., Uquillas, J.A., Akbari, M., Bertassoni, L.E., Cha, C., Camci-Unal, G., Dokmeci, M.R., Peppas, N.A., Khademhosseini, A. 25th Anniversary Article: Rational Design and Applications of Hydrogels in Regenerative Medicine Advanced Materials 26 (1) 2014: pp. 85-123.

https://doi.org/10.1002/adma.201303233

11. Geckil, H., Xu, F., Zhang, X.H., Moon, S.J., Demirci, U. Engineering Hydrogels as Extracellular Matrix Mimics Nanomedicine 5 (3) 2010: pp. 469-484. https://doi.org/10.2217/nnm.10.12

12. Tibbitt, M.W., Anseth, K.S. Hydrogels as Extracellular Matrix Mimics for 3D Cell Culture Biotechnology and Bioengineering 103 (4) 2009: pp. 655-663. https://doi.org/10.1002/bit.22361

13. Thakur, S., Govender, P.P., $\quad$ Mamo, M.A., Tamulevicius, S., Thakur, V.K. Recent Progress in Gelatin Hydrogel Nanocomposites for Water Purification and Beyond Vacuum 146 2017: pp. 396-408.

https://doi.org/ 10.1016/j.vacuum.2017.05.032

14. Yue, K., Trujillo-de, S.G., Alvarez, M.M., Tamayol, A., Annabi, N., Khademhosseini, A. Synthesis, Properties, and Biomedical Applications of Gelatin Methacryloyl (GelMA) Hydrogels Biomaterials 73 2015: pp. 254-271. https://doi.org/10.1016/j.biomaterials.2015.08.045

15. Kolambkar, Y.M., Dupont, K.M., Boerckel, J.D., Huebsch, N., Mooney, D.J., Hutmacher, D.W., Guldberg, R.E. An Alginate-based Hybrid System for Growth Factor Delivery in the Functional Repair of Large Bone Defects Biomaterials 32 (1) 2011: pp. 65-74. https://doi.org/10.1016/j.biomaterials.2010.08.074

16. Thakur, S., Arotiba, O. Synthesis, Characterization and Adsorption Studies of an Acrylic Acid-grafted Sodium Alginate-based $\mathrm{TiO}_{2}$ Hydrogel Nanocomposite Adsorption Science \& Technology 36 (1) 2017: pp. 458-477. https://doi.org/ 10.1177/0263617417700636

17. Zhang, Y.L., Tao, L., Li, S., Wei, Y. Synthesis of Multiresponsive and Dynamic Chitosan-based Hydrogels for Controlled Release of Bioactive Molecules Biomacromolecules 12 (8) 2011: pp. 2894-2901. https://doi.org/10.1021/bm200423f

18. Li, Z.Y., Su, Y.L., Haq, M.A., Xie, B.Q., Wang, D.J. Konjac Glucomannan/Polyacrylamide Bicomponent Hydrogels: Self-Healing Originating from SemiInterpenetrating Network Polymer 103 2016: pp. 146-151. https://doi.org/10.1016/j.polymer.2016.09.046

19. Thakur, S., Arotiba, O.A. Synthesis, Swelling and Adsorption Studies of a $\mathrm{pH}$-responsive Sodium AlginatePoly(Acrylic Acid) Superabsorbent Hydrogel Polymer Bulletin 2018: pp. 1-20. https://doi.org/10.1007/s00289-018-2287-0

20. Murphy, S.V., Skardal, A., Atala, A. Evaluation of Hydrogels for Bio-Printing Applications Journal of bBiomedical Materials Research Part A 101A(1) 2013: pp. $272-284$. https://doi.org/10.1002/jbm.a.34326

21. Katsuraya, K., Okuyama, K., Hatanaka, K., Oshima, R., Sato, T., Matsuzaki, K. Constitution of Konjac Glucomannan: Chemical Analysis and CNMR Spectroscopy Carbohydrate Polymers 53 (2) 2003: pp. 183-189. https://doi.org/10.1016/S0144-8617(03)00039-0

22. Li, Y.L., Deng, R.H., Chen, N., Pan, J., Pang, J. Review of Konjac Glucomannan: Isolation, Structure, Chain Conformation and Bioactivities Ling 1 (1) 2013: pp. 7. https://doi.org/10.12966/jsmr.07.03.2013
23. Yang, D., Yuan, Y., Wang, L., Wang, X., Mu, R., Pang, J., Xiao, J.B., Zheng, Y.F. A Review on Konjac Glucomannan Gels: Microstructure and Application International Journal of Molecular Sciences 18 (11) 2017: pp. 2250-2267. https://doi.org/10.3390/ijms18112250

24. Korkiatithaweechai, S., Umsarika, P., Praphairaksit, N., Muangsin, N. Controlled Release of Diclofenac from Matrix Polymer of Chitosan and Oxidized Konjac Glucomannan Marine drugs 9 (9) 2011: pp. 1649-1663. https://doi.org/10.3390/md9091649

25. Xu, Q., Huang, W.J., Jiang, L.B., Lei, Z.J., Li, X.Y., Deng, H.B. KGM and PMAA based pH-Sensitive Interpenetrating Polymer Network Hydrogel for Controlled Drug Release Carbohydrate Polymers 97 (2) 2013: pp. $565-570$. https://doi.org/10.1016/j.carbpol.2013.05.007

26. Jin, W.P., Song, R.K., Xu, W., Wang, Y.T., Li, J., Shah, B.R., Li, Y., Li, B. Analysis of Deacetylated Konjac Glucomannan and Xanthan Gum Phase Separation by Film Forming Food Hydrocolloids 48 2015: pp. 320-326. https://doi.org/org/10.1016/j.foodhyd.2015.02.007

27. Alonso-Sande, M., Teijeiro-Osorio, D., RemuñánLópez, C., Alonso, M.J. Glucomannan, a Promising Polysaccharide for Biopharmaceutical Purposes European Journal of Pharmaceutics \& Biopharmaceutics Official Journal of Arbeitsgemeinschaft Für Pharmazeutische Verfahrenstechnik E V 72 (2) 2009: pp. 453-462. https://doi.org/10.1016/j.ejpb.2008.02.005

28. Kang, K.H., Hockaday, L.A., Butcher, J.T. Quantitative Optimization of Solid Freeform Deposition of Aqueous Hydrogels Biofabrication 5 (3) 2013: pp. 035001-035013. https://doi.org/10.1088/1758-5082/5/3/035001

29. Dong, S.L., Han, L., Du, C.X., Wang, X.Y., Li, L.H., Wei, Y. 3D Printing of Aniline Tetramer-GraftedPolyethylenimine and Pluronic F127 Composites for Electroactive Scaffolds Macromol Rapid Commun 38 (4) 2017: pp. 1600551. https://doi.org/10.1002/marc.201600551

30. Diba, M., Wang, H., Kodger, T.E., Parsa, S., Leeuwenburgh, S.C. Highly Elastic and Self-Healing Composite Colloidal Gels Advanced Materials $29(11)$ 2017: pp. $1604672-1604678$. https://doi.org/10.1002/adma.201604672

31. Thakur, S., Pandey, S., Arotiba, O.A. Development of a Sodium Alginate-based Organic/Inorganic Superabsorbent Composite Hydrogel for Adsorption of Methylene Blue Carbohydr Polym 153 2016: pp. 34-46. https://doi.org/10.1016/j.carbpol.2016.06.104

32. Mandal, B.B., Kapoor, S., Kundu, S.C. Silk Fibroin/Polyacrylamide Semi-Interpenetrating Network Hydrogels for Controlled Drug Release Biomaterials 30 (14) 2009: pp. 2826-2836. https://doi.org/10.1016/j.biomaterials.2009.01.040

33. Zhang, Y., Tse, C., Rouholamin, D., Smith, P.J. Scaffolds for Tissue Engineering Produced by Inkjet Printing Central European Journal of Engineering 2 (3) 2012: pp. 325-335. https://doi.org/10.2478/s13531-012-0016-2

34. He, Y., Yang, F.F., Zhao, H.M., Gao, Q., Xia, B., Fu, J.Z. Research on the Printability of Hydrogels in 3D Bioprinting Scientific Reports 6 (6) 2016: pp. 29977-29989. 